Experimental Research

\title{
Enhancing of methotrexate penetration into the brain via the operation of indirect nonanastomotic by-pass technique (encephalo-myo-synangiosis) in rats
}

\author{
Cengiz Çokluk*a , Meftun Ünsal ${ }^{\mathrm{b}}$, Kerameddin Aydına, Abdulkerim Bedir \\ ${ }^{a}$ Department of Neurosurgery, Medical Faculty, Ondokuz Mayis University, Samsun, Turkey \\ ${ }^{b}$ Department of Chest Medicine, Medical Faculty, Ondokuz Mayis University, Samsun, Turkey \\ ${ }^{c}$ Department of Biochemistry, Medical Faculty, Ondokuz Mayis University, Samsun, Turkey
}

\begin{tabular}{|c|c|}
\hline \multicolumn{2}{|c|}{ ARTICLE INFO } \\
\hline \multicolumn{2}{|c|}{ Article History } \\
\hline Received & $31 / 03 / 2011$ \\
\hline Accepted & $05 / 04$ / 2011 \\
\hline
\end{tabular}

\section{* Correspondence to:}

Cengiz Çokluk

Ondokuz Mayis University,

Medical Faculty,

Department of Neurosurgery,

Samsun, Turkey

e-mail: cengizcokluk@yahoo.com

\section{Keywords:}

Nonanastomotic by-pass

Methotrexate

Brain parenchyma

Encephalo-myo-synangiosis

Brain Operation

Indirect by-pass

\begin{abstract}
The aim of this experimental study was to investigate the penetration of methotrexate into the brain parenchyma after indirect nonanastomotic by-pass surgery (encephalomyo-synangiosis) in rats. The fourteen rats were divided into two groups as control and operated rats. Control rats were not operated but received methotrexate treatment $(50 \mathrm{mg} /$ $\mathrm{kg}$ ). The operation of indirect nonanastomotic by-pass was done on operated group. After four weeks from the operation, methotrexate $(50 \mathrm{mg} / \mathrm{kg}$ ) was injected via intravenous route one hour before the decapitation. Parenchymal methotrexate level was estimated by using biochemical methods. The mean methotrexate level in the brain parenchyma of control group was estimated as $1.16 \pm 0.93 \mu \mathrm{mol} / \mathrm{L}$. The mean methotrexate level in the brain parenchyma of operated group was found as $2.5 \pm 0.98 \mu \mathrm{mol} / \mathrm{L}$. The operation of pial synangiosis enhanced the penetration of methodrexate into the brain parenchyma in comparison with unoperated subjects. The differences between these two groups were statistically meaningful $(\mathrm{p}=0.0221)$. In this experimental study, we used the operation of encephalo-myo-synangiosis (EMS) for enhancing of the penetration of methotrexate into the brain. According to our results the operation of EMS enhanced the penetration of methotrexate into the brain parenchyma in the rats.

J. Exp. Clin. Med., 2011; 28:17-21
\end{abstract}

\section{Introduction}

Despite much advancement in the innovation and using of antineoplastic agents, the chemotherapeutic treatment of central nervous system (CNS) tumors and its metastatic involvement, still have some controversies because of blood brain barrier. This barrier is responsible for poor penetration of chemotherapeutic drugs into the nervous system (Rall, 1965; Griffin and Rasey, 1977).

Methotrexate is one of the antineoplastic agents. It is used for many tumors. The penetration of methotrexate into the brain parenchyma is difficult because of poor blood brain barrier permeability. For this reason, in the chemotherapeutic treatment of malignant lesions seated within the neural parenchyma, the administration of intratecal methotrexate injection is generally used in clinical practice (Orlando et al., 2002; Gao and Jiang, 2006). Insertion of a reservoir for injection of agents or local chemo-agent treatment may be preferred in the other alternative choices (Orlando et al., 2002).

In this experimental study, we used nonanastomotic indirect by-pass surgery using temporal muscle (EMS) in the rats for enhancement of methotrexate penetration into the brain parenchyma. It is known that extracranial capillary system doesn't have the tight junctions between endothelial cells mainly represented from the blood brain barrier. The newly forming capillaries originated from extracranial vessels structurally will not have endothelial tight junctions such as intracranial vessels. We hypothesized that these new capillaries may be used for chemotherapeutic agents delivering into the brain tissue after indirect nonanastomotic by-pass surgery. This experimental study was designed to investigate the efficacy of EMS in the enhancing of methotrexate penetration into the brain in rats.

\section{Materials and Methods}

The present study was approved by the local animal care committee at Medical Faculty of Ondokuz Mayis University (Local Ethical Committee).

Fourteen male Sprague-Dawley rats, each weighing between 280 and 320 gram, were used for this study. The rats were divided into two groups as control (Group I) and EMS (Group II). In this experimental study, Methotrexate (Metotreksat Atafarm Drug co., Istanbul, Turkey) was used in the evaluation of the efficacy of EMS on the enhancing of the penetration of chemotherapeutic drugs into the brain. 
Methotrexate formerly known as amethopterin is an antimetabolite and antifolate drug used in treatment of cancer and autoimmune diseases. It acts by inhibiting the metabolism of folic acid. The chemical formula of methotrexate is $\mathrm{C} 20 \mathrm{H} 22 \mathrm{~N} 8 \mathrm{O} 5$. The molecular mass was estimated as 454.44 $\mathrm{g} / \mathrm{mol}$. The systematic name of methotrexate is as follows; (2s)-2-[(4-\{[(2, 3-diamino-7, 8-dihidropteridin-6yl) methyl $]$ (methyl) amino phenyl) formamido] pentanedioic acid. Its metabolism is mainly hepatic. The half life was found as 3 and 15 hours but its half life is mainly dose dependent. Its excretion from the body is mainly renal route (48-100\%). It can be used oral, intravenous, intramuscular, subcutaneous and intrathechal routes.

The experimental protocol for Group I rats was as follows; Prior to procedure the animals were allowed free access to food and water in their cages with a 12 hours light/dark cyscle and a temperature of $22{ }^{\circ} \mathrm{C}$. Before starting the procedure for Group I the rats were anesthetized with ketamine $(4 \mathrm{mg} / 100 \mathrm{gr})$ and xylazine $(1.5 \mathrm{mg} / 100 \mathrm{gr})$ administered intramuscularly. Hematocrit, $\mathrm{pH}, \mathrm{PO}_{2}$ and $\mathrm{PCO}_{2}$ and blood pressure were monitored during the procedure via the femoral artery and vein. Rectal temperature was maintained at $37{ }^{\circ} \mathrm{C}$ with heating a pad throughout the procedure. After establishing optimum physiological conditions for experimental procedure, methotrexate was given intravenous route. Femoral vein was used for the intravenous route. One hour later from finishing the intravenous methotrexate injection, the rats were decapitated with guillotine technique under the general anesthesia. The right hemispheres of the brains were removed and placed in test tubes. The brains were kept up to $-80{ }^{\circ} \mathrm{C}$ until the procedures of biochemical measurements.

The experimental protocol for Group II (EMS) rats was as follows; prior to surgery the animals were allowed free access to food and water in their cages with a 12 hour light/dark cycle and a temperature of $22{ }^{\circ} \mathrm{C}$. The rats were anesthetized with ketamine (4 mg/100 gr) and xylazine (1.5 mg /100 gr) administered intramuscularly. Hematocrit, $\mathrm{pH}, \mathrm{pO}_{2}$ and $\mathrm{pCO}_{2}$ and blood pressure were monitored during surgery via the femoral artery and vein. Rectal temperature was maintaned at $37{ }^{\circ} \mathrm{C}$ with heating a pad throughout the operation. Each animal was placed in the semi-lateral position on the operating table. A midline straight skin incision was done on the scalp from anterior to posterior direction (Fig.1A and B).

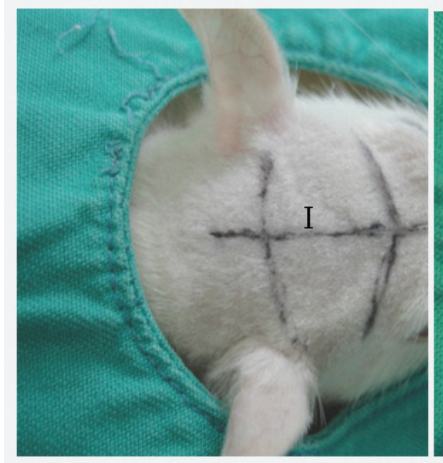

A

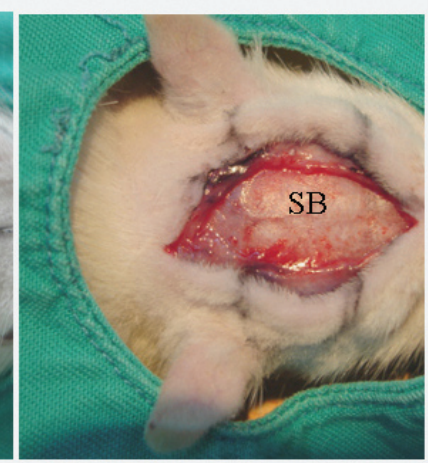

B
Fig. 1. A.The planned skin incision is shown (I: Incision). B. After per forming the skin incision the skull bone is appeared (SB: Skull bone).
The temporalis muscle was cut on the superior and posterior margins from the attachment to the bone and was retracted to the inferior and posterior direction. After exposing the posterior part of the zygomatic arc, temporal and parietal bone was drilled out (Fig. 2A and B).

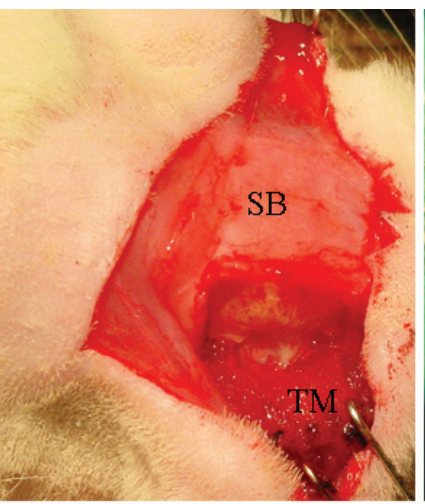

A

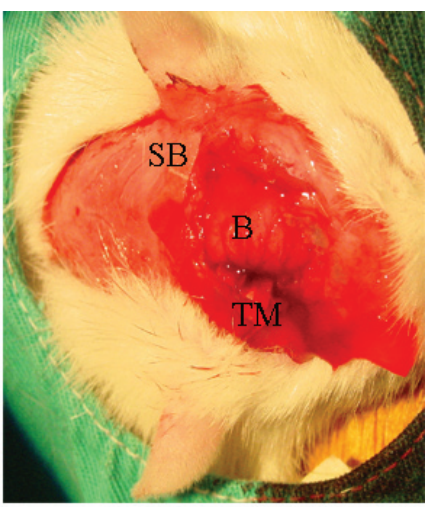

$\mathrm{B}$
Fig. 2. A.Dissection and separation of temporal muscle from skull bone (SB: Skull bone, TM: Temporal muscle). B. Opening of the skull bone to exposing of dura mater and brain parenchyma (SB: Skull bone, B: Brain, TM: Temporal muscle).

The dura mater was exposed. An anterior-posterior and superior-inferior linear dural incision was done for exposing of the pia-arachnoid membrane by using the tip of a 21 gauge tuberculin needle. After opening the dura mater, temporal muscle was faced over the pial surface. After this procedure skin was closed with $4 / 0$ nylon suture.

After the operation, the rats were recovered in their cages. After four weeks from the operation, they were anesthetized with same procedure described previously. Following the anesthesia and related monitoring principles, all rats received an intravenous injection of $50 \mathrm{mg} / \mathrm{kg}$ methotrexate. One hour later, they were decapitated and right hemispheres of the brains were removed and placed in test tubes. The operated site of the brain was shown in Fig. 3A and B.

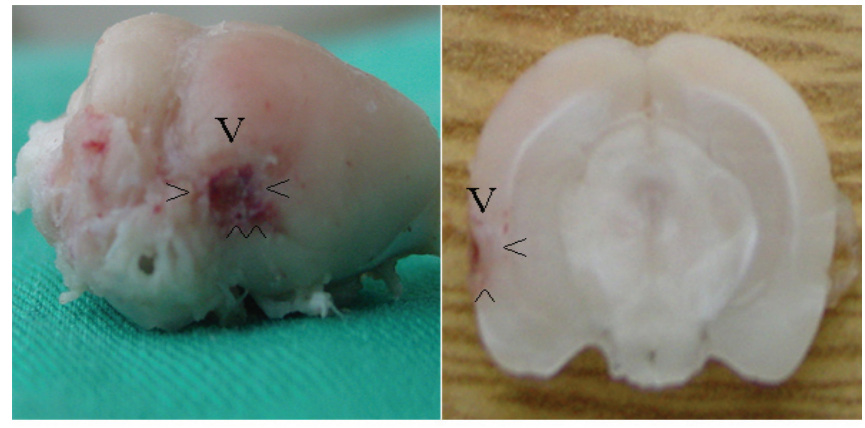

A

B

Fig. 3. A. After removing of the brain tissue the operative site is shown (arrows show the operative site) B. The operative site is shown in sliced brain (arrows show operative site).

As same with Group I rats, the brains were kept up to -80 ${ }^{\circ} \mathrm{C}$ until the procedures of biochemical measurements. The rat brains were stored in the same shelf of the deep freeze machine. The following day, all the specimens were transferred to the biochemistry laboratory within a cold keeper bag. The brain tissues were pulverized with liquid nitrogen. 
The weights of brain tissues were estimated.

The micro-total protein levels of supernatants were estimated by using a Roche COBAS INTEGRA 800 machine with Total Protein Urine /CSF Gen 3 (TPUC3) kit using the turbid metric method. HPLC.

Levels of methotrexate in brain were estimated by

All the measured values are presented in Table 1 and 2, and were analyzed statistically.

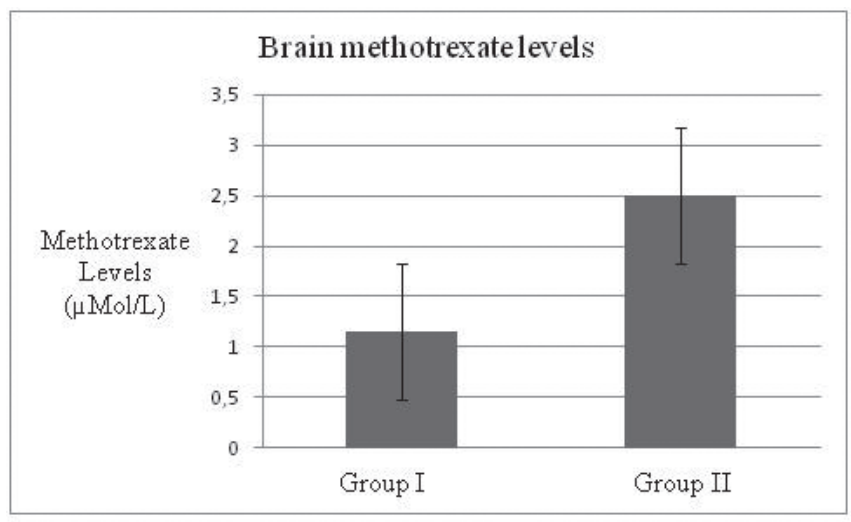

Fig. 4. Brain methotrexate levels in Group I and Group II.

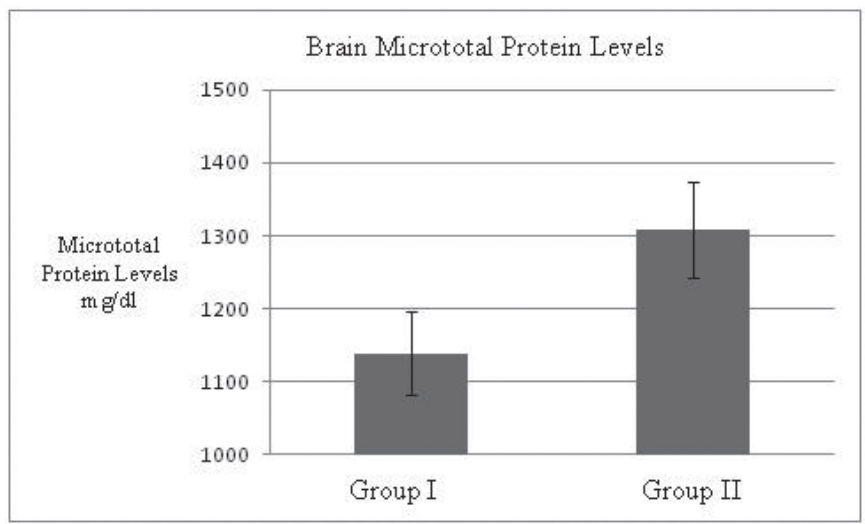

Fig. 5. Brain micrototal protein levels in Group I and Group II.

The chi square test was used to determine the significance of differences among the groups and p values of less than .05 was considered statistically significant.

\section{Results}

Methotrexate (Metotreksat Amphar b.v. Flakon), the commercially available antineoplastic drug was used for this experiment. After calculating the appropriate dose, the drug was injected through the rat femoral vein. Before and immediately after the injection, the blood pressure and heart rate were measured via a catheter inserted into the femoral artery. Blood pressure and heart rate in rats did not change significantly after the injections of methotrexate.

Postoperative daily clinical examinations were performed in all rats. There was no operative mortality and infection. Postoperative clinical examinations were noted as normal in all rats.

During removing the rat brains, the operation sites in EMS rats were inspected with gross-microscopically. There was no infection and hematoma finding in any rats. The in- direct by-pass flap attached to the brain surface in all EMS rats.

Two different parameters were measured in the evaluation of the results of this experimental study. One of these parameters was the level of methotrexate in the brain. Another of them was the level of micro-total protein in the brain.

The level of methotrexate in Group I (Control) was estimated as $1.16 \pm 0.93 \mu \mathrm{mol} / \mathrm{L}$. In the other hand the level of methotrexate in Group II (EMS) rats was estimated as $2.5 \pm$ $0.98 \mu \mathrm{mol} / \mathrm{L}$. The levels of methotrexate were shown in Table 1 . It was found the presence of $54 \%$ difference between Group I and Group II in terms of methotrexate levels (Fig. 4). The differences between these two groups were statistically significant $(\mathrm{p}=0.0221)$.

The mean micro total protein level was estimated as $1044.3 \pm 88.67 \mathrm{mg} / \mathrm{dl}$ in Group I. In Group II, the mean micrototal protein level was found as $1308.6 \pm 185.9 \mathrm{mg} / \mathrm{dl}$. There is $21 \%$ difference between Group I and Group II (Fig. 5). The micrototal protein levels were shown in Table 2. The differences between these two groups were statistically significant $(\mathrm{p}=0.0113)$.

\section{Discussion}

Tumor lesions located in the CNS system can be mainly divided into two main groups as primary and secondary tumors. The term of primary tumors are used for describing of the tumors primarily originated from the nervous system cells. The term of secondary tumors are used for metastatic lesions. Primary brain tumors and secondary brain metastasis represent an important cause of morbidity and mortality in adults and child. Surgery, chemotherapy and radiotherapy are the treatment methods.

Table 1. The levels of methotrexate in Group I and Group II.

\begin{tabular}{|l|l|l|}
\hline $\begin{array}{l}\text { Rat } \\
\text { number }\end{array}$ & $\begin{array}{c}\text { Group I (Control) } \\
\text { (MTX level } \mu \mathrm{mol} / \mathrm{l})\end{array}$ & $\begin{array}{l}\text { Group II (Pial } \\
\text { Synangiosis) (MTX } \\
\text { Level } \mu \mathrm{mol} / \mathrm{l})\end{array}$ \\
\hline 1 & 0.55 & 4.26 \\
\hline 2 & 0.379 & 2.914 \\
\hline 3 & 1.413 & 2.138 \\
\hline 4 & 0.786 & 1.514 \\
\hline 5 & 1.412 & 1.608 \\
\hline 6 & 0.528 & 1.978 \\
\hline 7 & 3.068 & 3.105 \\
\hline MEAN \pm SD & $1.16 \pm 0.93$ & $2.5 \pm 0.98$ \\
\hline
\end{tabular}

Table 2. The level of micrototal proteins in the rats

\begin{tabular}{|l|l|l|}
\hline $\begin{array}{l}\text { Rat } \\
\text { number }\end{array}$ & $\begin{array}{c}\text { Group I (Control) } \\
(\mathrm{mg} / \mathrm{dl})\end{array}$ & $\begin{array}{l}\text { Group II (Pial } \\
\text { synangiosis }) \\
(\mathrm{mg} / \mathrm{dl})\end{array}$ \\
\hline 1 & 950 & 1260 \\
\hline 2 & 1170 & 1380 \\
\hline 3 & 950 & 1000 \\
\hline 4 & 1090 & 1140 \\
\hline 5 & 1060 & 1420 \\
\hline 6 & 970 & 1530 \\
\hline 7 & 1120 & 1430 \\
\hline MEAN \pm SD & $1138.6 \pm 88.67$ & $1308.6 \pm 185.9$ \\
\hline
\end{tabular}


Although chemotherapy had been defined as a treatment method for many years, there is no common consensus because of chemo sensitivity and the blood brain barrier. The presence of intact or partially intact blood brain barrier is the main limitation of chemotherapy in these cases. Some different methods had been defined for diversion and/or penetration of blood brain barrier. Some of these methods are used together with systemic chemotherapy. Some others are used in focal chemotherapy treatment methods. The term of focal chemotherapy treatment methods includes the direct delivery of chemotherapeutic agents to the central nervous system. These methods include the use of Ommaya reservoirs, intrathecal injections (Orlando et al., 2002), intra-arterial injections (Cloughesy et al., 1997; Newton et al., 2003), or high-dose systemic therapy with some agents such as methotrexate (Lassman et al., 2006). Another method is to give chemotherapy drugs with some agents that inhibit the drug transport of the blood brain barrier such as mannitol.

Blood brain barrier had been defined by Deekens and Loscher (Deeken and Löscher, 2007). Endothelial tight junctions and glial cellular barrier restrict the passage of some materials into the brain parenchyma. Extracellular matrix, pericytes, and astrocytical foot processes further mediate the specific permeability of blood brain barrier. The main blood brain barrier is the lack of fenestrated endothelial cells and the presence of tight junctions in the brain capillary vasculature (Bellavance et al., 2008).

The passing of chemotherapeutic agents across the blood brain barrier is the main obstacle in the medical treatment of primary and secondary brain tumors. It is necessary the diversion and/or facilitation procedures for sufficient and effective delivering of the chemotherapeutic agents to the tumor cells and interstitial space of tumor tissue seated deep in the brain (Kemper et al., 2004; Deeken and Löscher, 2007). For this purpose we developed a new animal procedure for diversion of blood brain barrier and facilitation of the penetration of the chemotherapeutic agents into the brain. In our procedures we used EMS for revascularization (Karasawa et al., 1977; Yoshioka and Tominaga, 1998).

The technique of EMS had been described treating of cerebral vascular insufficiency such as the moya moya disease and other vascular occlusive pathologies (Karasawa et al., 1977; Yoshioka and Tominaga, 1998). In this procedure, extracranial tissues is mobilized with protection of their vascular feeders and exposed to pia mater. For this purpose, the temporal muscle is frequently used. Following this procedure the micro-capillary vasculature of extracranial structures in- vades the very delicate membrane of pial mater to reach down the interstitial and extracellular matrix space of nervous system for developing of new vascularization tree. Yoshioka et al. reported the efficacy of EMS on cerebral revascularization not only in patients with adult moyamoya disease, but also in elderly patients with atherosclerotic ischemic disease (Yoshioka and Tominaga, 1998).

We hypothesized that the rats underwent EMS operation will have more methotrexate concentration in their brain tissue than those of unoperated control rats. In our experiment following the EMS operation the rats were housed in their cages keeping natural environmental conditions for four weeks. This time period is necessary for proliferation, developing and generation of new micro capillary vasculature. Following this standby period, all rats were re-anesthetized with same procedure. methotrexate was prepared and injected via venous route for reaching of fast and effective blood methotrexate levels. Following this procedure the rats were hemodinamically stabilized and monitorized for one hour with optimal environmental condition in where described previously in methods section. After one hour period, the rats decapitated under the effect of general anesthesia. The brains of all rats were removed. In the biochemical evaluation, we used two different parameters; micro-total protein levels of the brain and brain methotrexate levels. The height of the brain protein levels in EMS rats was found as $21 \%$ higher than those of control rats. The differences between EMS and control rats were statistically significant $(\mathrm{p}=0.0113)$. There is close relationship between brain protein levels and the permeability of blood-brain barrier. Increased brain protein levels can be explained with increased permeability of blood brain barrier. The most important part of this study was to measure the level of methotrexate in the brain. The systemic use of methotrexate is not common in brain tumors because of low blood brain barrier permeability. Group I rats had a low brain methotrexate levels $(1.16 \pm 0.93 \mu \mathrm{mol} / \mathrm{L})$. The situation was different in Group II rats. EMS surgery enhanced the brain methotrexate levels in the ratio of $54 \%$. The differences between these two group was statistically significant $(\mathrm{p}=0.0221)$.

\section{Conclusion}

The results of this experimental study show us that EMS has the increasing effect on the methotrexate permeability to the brain in rats. After brain tumor removal indirect nonanastomotic by-pass procedures may be performed to enhance the chemotherapeutic permeability to the brain.

\section{REFERENCES}

Bellavance, M.A., Blanchette, M., Fortin, D. 2008. Recent advances in blood-brain barrier disruption as a CNS delivery strategy. AAPS J. 10, 166-177

Cloughesy, T.F., Gobin,Y.P., Black, K.L., Vinuella, F., Taft, F., Kadkhoda, B., Kabbinavar, F. 1997. Intra-arterial carboplatin chemotherapy for brain tumors: a dose escalation study based on cerebral blood flow. J. Neurooncol. 35, 121-131

Deeken, J.F., Löscher, W. 2007. The blood-brain barrier and cancer: transporters, treatment, and Trojan horses. Clin. Cancer Res. 13, 16631674

Gao, K., Jiang, X. 2006. Influence of particle size on transport of methotrexate across blood brain barrier by polysorbate 80-coated polybutylcy anoacrylate nanoparticles. Int. J. Pharm. 310, 213-219

Griffin, T.W., Rasey, J.S., Bleyer, W.A. 1977. The effect of photon irradiation on blood brain barrier permeability to methotrexate in mice. Cancer. 40, 1109-1111

Karasawa, J., Kikuchi, H., Furuse, S., Sakaki, T., Yoshida, Y. 1977. A surgical treatment of moyampya disease. Encephalo-myo-synangiosis. Neurol. Med. Chir 17, 29-37

Kemper, E.M., Boogerd, W., Thuis, I., Beijnen, J.H., van Tellingen, O. 2004. Modulation of the blood-brain barrier in oncology: therapeutic opportunities for the treatment of brain tumours? Cancer Treat. Rev. 30, 415-423 
Lassman, A.B., Abrey, L.E., Shah, G.D., Panageas, K.S., Begemann, M., Malkin, M.G., Raizer, J.J. 2006. Systemic high-dose intravenous methotrexate for central nervous system metastases. J. Neurooncol. 78, 255-260

Newton, H.B., Slivka, M.A.,Volpi, C., Bourekas, E.C., Christoforidis, G.A., Baujan, M.A., Slone, W., Chakeres, D.W. 2003. Intra-arterial carbo platin and intravenous etoposide for the treatment of metastatic brain tumors. J. Neurooncol. 61, 35- 44

Orlando, L., Curigliano, G., Colleoni, M., Nole, F., Ferretti, G., Masci, G., Peruzotti, G., Minchella, I., Intra, M., Veronesi, P., Viale, G., Gol dhirsch, A. 2002. Intrathecal chemotherapy in carcinomatous meningitis from breast cancer. Anticancer Res. 22, $3057-3059$ Rall, P.D. 1965. Experimental studies oft he blood brain barrier. Cancer Res. 25, 1572-1577

Yoshioka, N., Tominaga, S. 1998. Cerebral revascularization using muscle free flap for ischemic cerebrovascular disease in adult patients. Neurol. Med. Chir 38, 464-468 